UCKL-ID-108241

\title{
The LLNL Configurable Seismic Monitoring System Final Report
}

\author{
K. K. Nakanishi, J. J. Zucca, B. C. Bowman, D. W. Ewert, \\ P. E. Harben, S. P. Jarpe, C. A. Johnston, and D. W. Rock
}

September 23, 1991

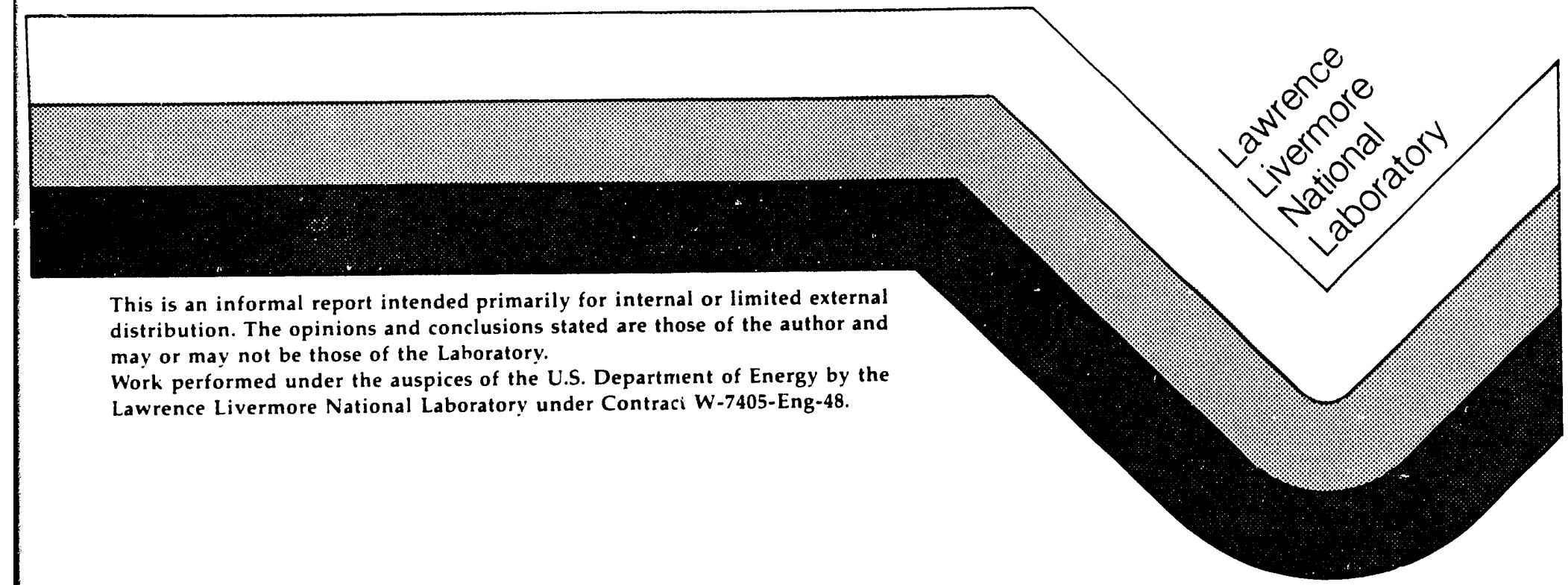




\section{DISCLAIMER}

This document was prepared as an acccount of work sponsored by an agency of the United States Government. Neither the United States Government nor the University of California nor any of their employees, makes any warranty, express or implied, or assumes any legal liability or responsibility for the accuracy, completeness, or usefulness of any information, apparatus, product, or process disclosed, rr represents that its use would not infringe privately own rights. Reference herein to any specific commercial products, process, or service by trade name, trademark, manufacturer, or otherwise, does not necessarily constitute or imply its endorsement, recommendation, or favoring by the United States Government or the University of California. The view's and opinions of author, expressed herein do not necessarily state or reflect those of the United States Government or the University of California, and shall not be used for advertising or product endorsement purposes.

This report has been reproduced directly from the best available copy.

Available to DOE and DOE contractors from the Office of Scientific and Technical Information P.O. Box 62, Oak Ridge, TN 37831

Prices available from (615) 576-8401, FTS 626-8401

Avaica. ${ }^{2}$ le to the public from the National Tecnnical Information Service

U.S. Department of Commerce 5285 Port Royal Rd. Springfield, VA 22161 


\title{
The LLNL Configurable Seismic Monitoring System Final Report
}

\author{
K. K. Nakanishi, J. J. Zucca, B. C. Bowman, D. W. Ewert, \\ P. E. Harben, S. P. Jarpe, C. A. Johnston, and D. W. Rock
}

\begin{abstract}
Summary
Lawrence Livermore National Laboratory (LLNL) developed and tested a portable, hiqh-quality seismic recording system for monitoring underground nuclear explosions. This configurable seismic monitoring system (CSMS) was designed to satisfy the designated seismic station (DSS) requirements for instrumentation to be deployed in a vault as set forth in the protocol of the Threshold Test Ban Treaty (TTBT). As a proof-of-concept test under treaty monitoring conditions, two configurations of this system were built, and each was deployed at a different seismic station to record the Nevada Test Site (NTS) event Bexar. Both configurations successfully recorded this event, demonstrating the flexibility and the operation of the basic design.
\end{abstract}

\section{Introduction}

Designing portable data acquisition systems, such as the CSMS, is part of LLNL's ongoing effort to improve the seismic monitoring technologies used to verify compliance with underground nuclear test limitation treaties. This class of instruments is designed to include the following characteristics:

1. The components are commercially available, off-the-shelf products.

2. The system is modular and can accommodate various sensors, digitizers, telemetry, and data archival equipment.
3. The system is simple to install and operate.

4. The system is robust and field tested.

5. The design and operating characteristics are documented.

6. The system is easy to pack and can be shipped by commercial carriers.

Assembling the two test versions of the CSMS required integrating the components, developing the software to link these components, and providing data acquisition, processing, signal viewing, and archiving capabilities.

\section{Designated Seismic Stations}

The protocol to the Threshold Test Ban Treaty gives the monitoring country the right to place three temporary designated seismic stations in the territory of the testing country, when the planned yield of the test is above 50 kilotons. The equipment includes sensors, amplifiers, filters, digitizers, recording equipment, cable for connecting the components, the ability to control and calibrate the sensors and recorders, a means of recording universal time, and equipment to process the data, monitor data quality, and provide the capability to display, store, and copy the data recorded. Three implicit conditions are: (1) the equipment must be reliable and, since the system may be operated by nonspecialists, (2) it must also be simple to set up, and (3) its software must be "user-friendly," and well-documented. 


\section{System Description}

We assembled two portable seismic station models. The first model was an adaptation of the LLNL Local Seismic Network technology (Zucca, 1988; Peterson et al., 1988) in which we replaced radio telemetry with a direct wire line (for a detailed discussion of this model, see Harben et al., 1990). In this paper, we discuss the second model, which consisted of two configurations (one with a 24-bit digitizer and the other with a 16-bit digitizer) that we designed explicitly for DSS applications (Fig. 1). For a final proof-of-concept test, we deployed both configurations of the second model to record the NTS event Bexar (April 4, 1991); the version with the 16-bit digitizer was deployed at the Oklahoma Geophysical Observatory near Leonard, Oklahoma (the Tulsa deployment), and the version with the 24-bit digitizer was deployed at the Air Force Technical Applications Center (AFTAC) Pinedale Seismic Research Facility in Pinedale, Wyoming (the Pinedale deployment) (Figs. 2-4).

The CSMS units are modular and use commercially available, off-the-shelf components. Our component integration and software designs are flexible enough to allow the substitution of different components, depending on the requirements of a particular application. In the configuration selected for the DSS application, we use a broadband Guralp CMG-3ESP, three-component seismometer. The passband is set from 0.1 to $10 \mathrm{~Hz}$ with the high pass rolloff at $6 \mathrm{~dB} /$ octave and the low pass rolloff at $36 \mathrm{~dB} /$ octave. The digitizers process the seismometer output at 40 samples per second per component. Universal
Vault Subsystem

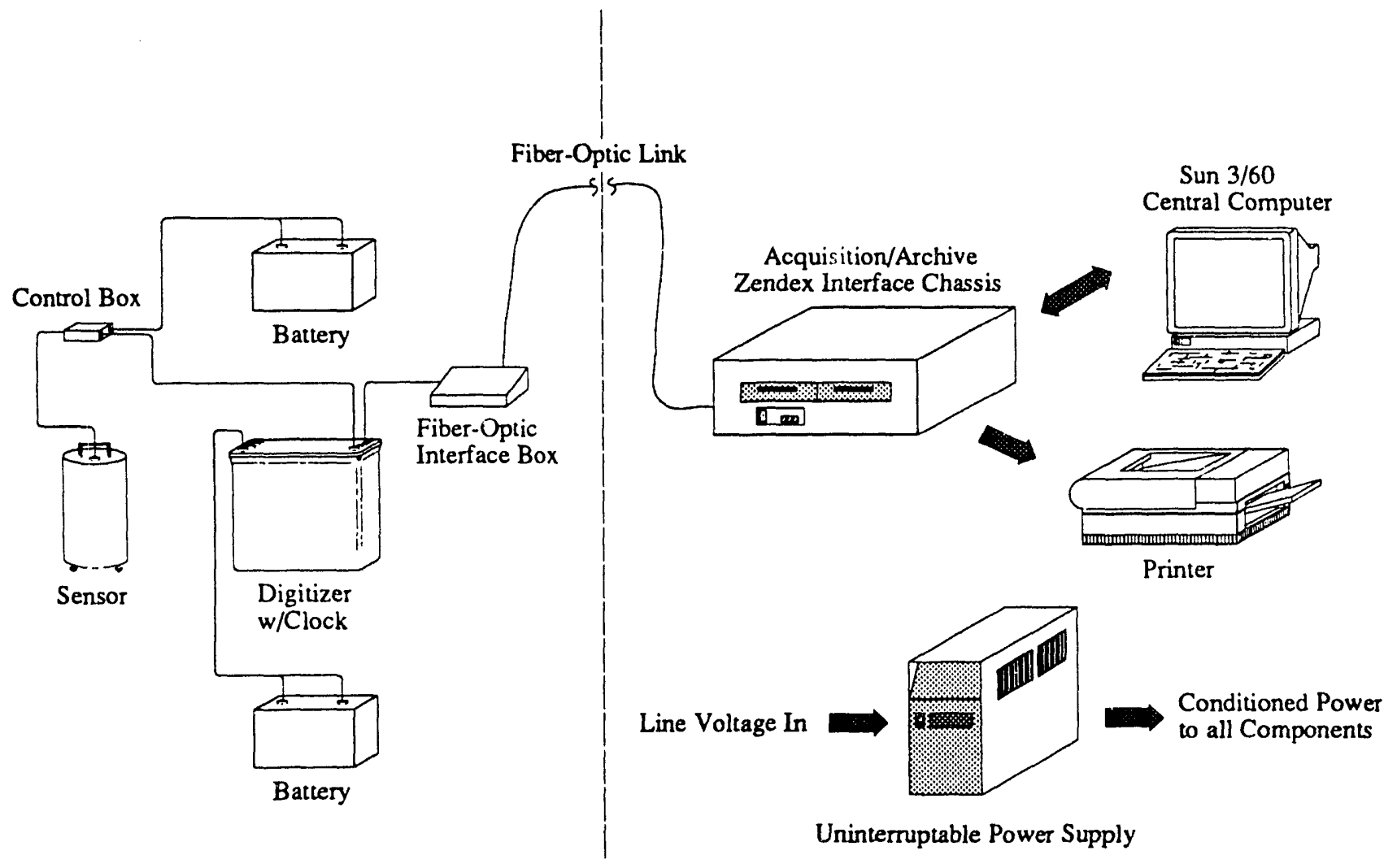

Figure 1. CSMS system configuration. 


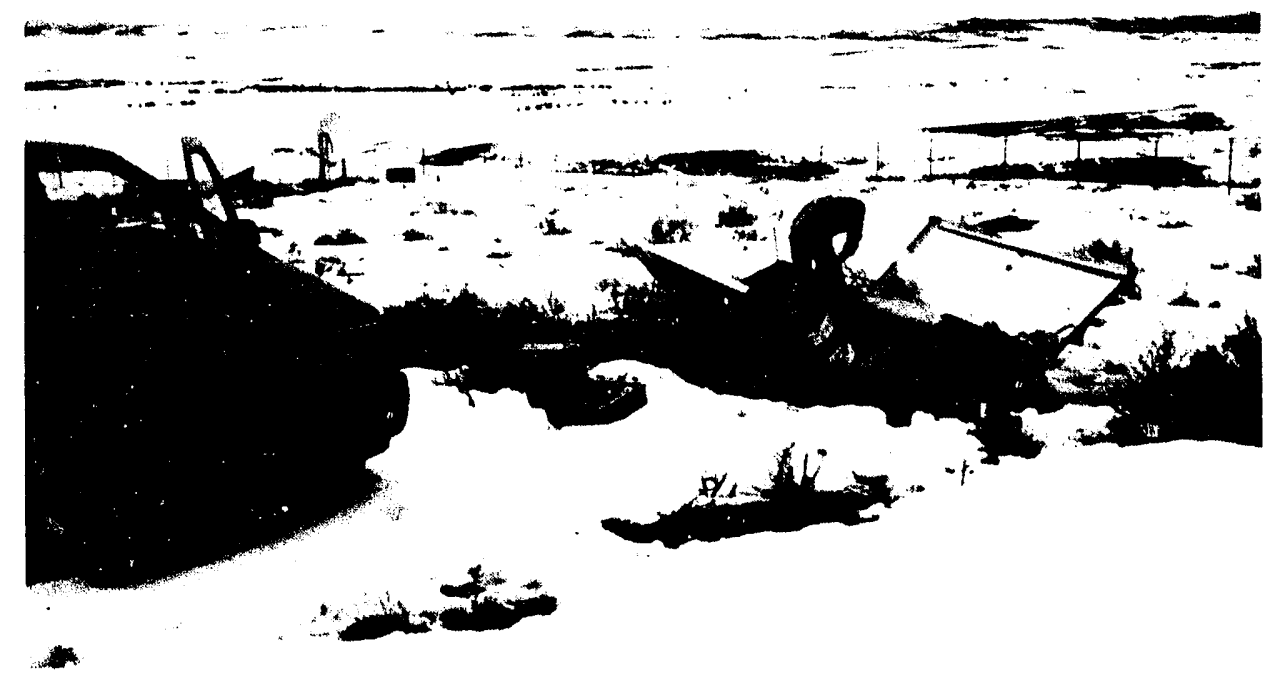

Figure 2. AFTAC Pinedale Seismic Research Facility in Wyoming. Entrance to the 13-meter-deep remote seismometer vault is in the foreground, and the entrance to the central recording vault is in the distance, near the satellite dish.

time is provided by the Omega standard. The seismometer and the digitizer are placed in a remote seismic vault, and the digitized signal is transmitted by fiber-optic cable to a central recording station.

The central station consists of a Sun $3 / 60$ computer workstation, an interface chassis, and a laser printer. The interface chassis' components were integrated by LLNL and include a 183-MB hard disk, a communications board, a SCSI host adaptor, and dual digital audio tape (DAT) drives. These dual drives allow the simultaneous recording of identical data to two tapes, as per TTBT protocol.

The communications board is a receiver for the digitized data from the fiber-optic link, and it buffers the incoming signals for the Sun $3 / 60$ computer. It is a 68010-based contrcller with 512kB RAM, and it makes high-speed asynchronous data transfers via the SCSI bus directly to the Sun workstation.

The primary functions of the CSMS software system are data acquisition, status monitoring,

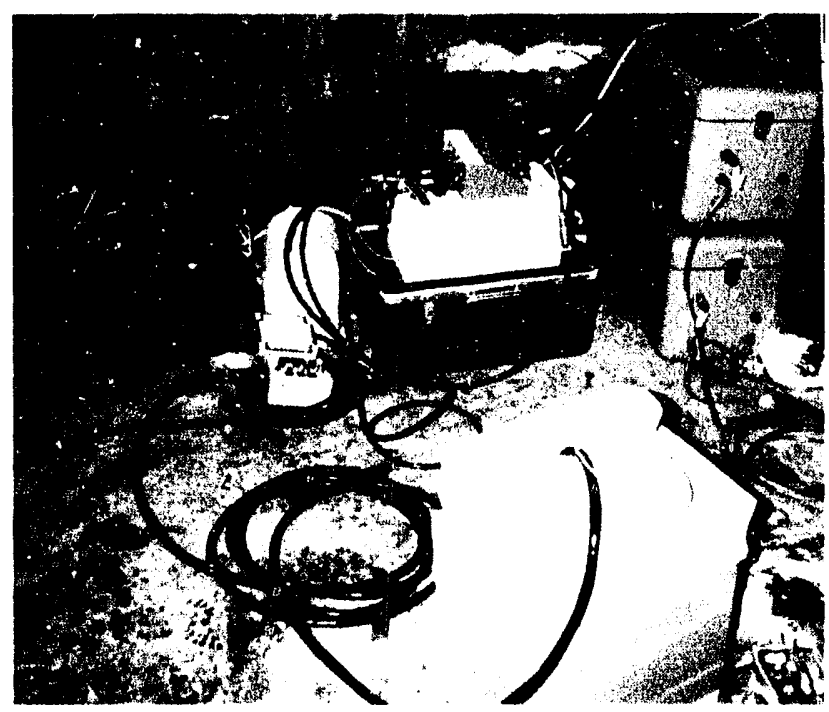

Figure 3. Remote seismometer vault at Pinedale. The Guralp sensor is in the foreground, enclosed in Styrofoam for thermal insulation, and the white box against the wall in the center is the digitizer. The fiber-optic cable interface, batteries, and connector cables are also shown. 


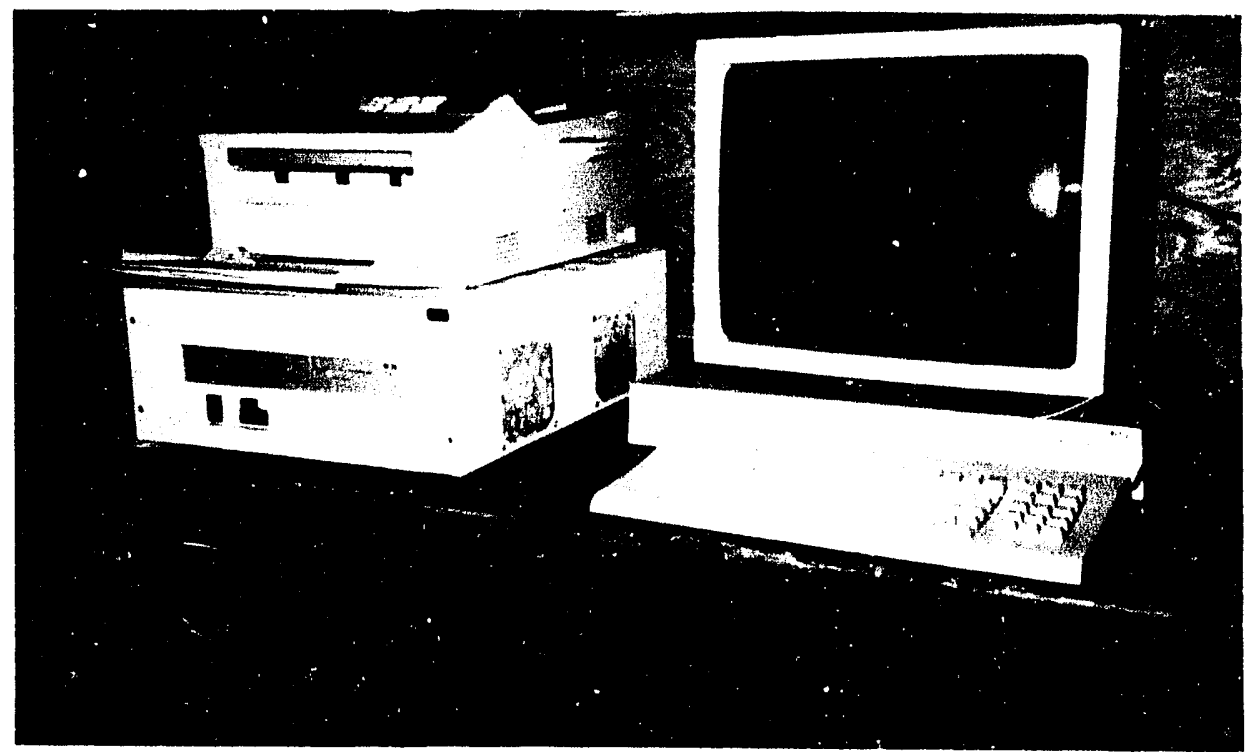

Figure 4. Central recording and processing facility at Pinedale. The Sun 3/60 computer is on the right, and the chassis containing the disks, DAT drives, and interface cards is on the left. The laser printer is on top of the interface chassis.

waveform monitoring, digitizer command and control, data archiving, and data analysis. The digitized signal is passed from the communications board to the Sun 3/60. Monitor programs provide continuous system statistics and a real-time display of the signals from all data channels. These displays allow the operator to monitor data quality during setup and calibration as well as during the experiment itself. The data are displayed, are stored to disk, and are finally archived to digital audio tape.

\section{Bexar Deployment}

To test and demonstrate the CSMS, we deployed the system at two sites to record the NTS event Bexar under treaty monitoring conditions.

At the Pinedale site, the remote seismic vault and the central recorder were separated by about 50 meters (Fig. 2). The remote station (consisting of the seismometer, the 24-bit digitizer, and the fiber-optic coupler) was located in a 13-meter-deep vault (Fig. 3). The central recording station was placed in a walk-in vault and consisted of the Sun 3/60 computer, communications board, disk, archive DAT drives, laser printer, and power line conditioner (Fig. 4). The recording vault was located about 20 meters from the DOE deployable seismic verification system (DSVS) station.

At the Tulsa site, the seismometer pier was in a 4-meter-deep vault, 63 meters from the Tulsa
DSS, and in the same geological formation. The vault equipment was identical to the Pinedale instrumentation with the exception of the 16-bit digitizer instead of the 24-bit digitizer used in Pinedale. The central recording equipment was located 15 meters away from the vault and was identical to the unit at Pinedale with the exception of the printer.

Figures 5 and 6 are unprocessed recordings of Bexar from Pinedale and Tulsa, respectively. The seismic traces for the CSMS and DSVS vertical broadband channels are shown in Fig. 7, and the corresponding amplitude spectra are shown in Fig. 8. A comparison of the waveforms and spectra indicate that the CSMS recorded data with fidelity equivalent to that of the DSVS. 


\section{Conclusions}

Lawrence Livermore National Laboratory assembled a seismic recording system designed to satisify the TTBT requirements for a designated seismic station. The CSMS developed and tested by LLNL is both flexible and integrates all of the functions specified in the TTBT. In addition, the system is reliable, easy to set up, and the software is user-friendly. The final deployment of the CSMS to record the NTS event Bexar under treaty monitoring conditions demonstraled that it is capable of fulfiling all of the functional and operational requirements for a portable, high-quality seismic recording system.

\section{Bibliography}

Harben, P. E., D. W. Rock, and R. C. Carlson (1990), The CSMS Poorboy Deployment: Seismic Recording in Pinedale, Wyoming, of the Bullion NTS Nuclear Test under the Verification Provisions of the new TTBT Protocol, Lawrence Livermore National Laboratory, Livermore, CA, UCRL-ID-104332.

Peterson, S. J., J. J. Zucca, D. W. Ewert, D. J. Moccia, D. W. Rock, and R. K. Stager (1988), The Configurable Seismic Monitoring System (CSMS): Local Seismic Network (LSN) Data Acquisition User's Manual Version 1.0, Lawrence Livermore National Laboratory, Livermore, CA, UCID-21174.

Zucca, J. J. (1988), The Configurable Seismic Monitoring System, Lawrence Livermore National Laboratory, Livermore, CA, LLL-TB-99.

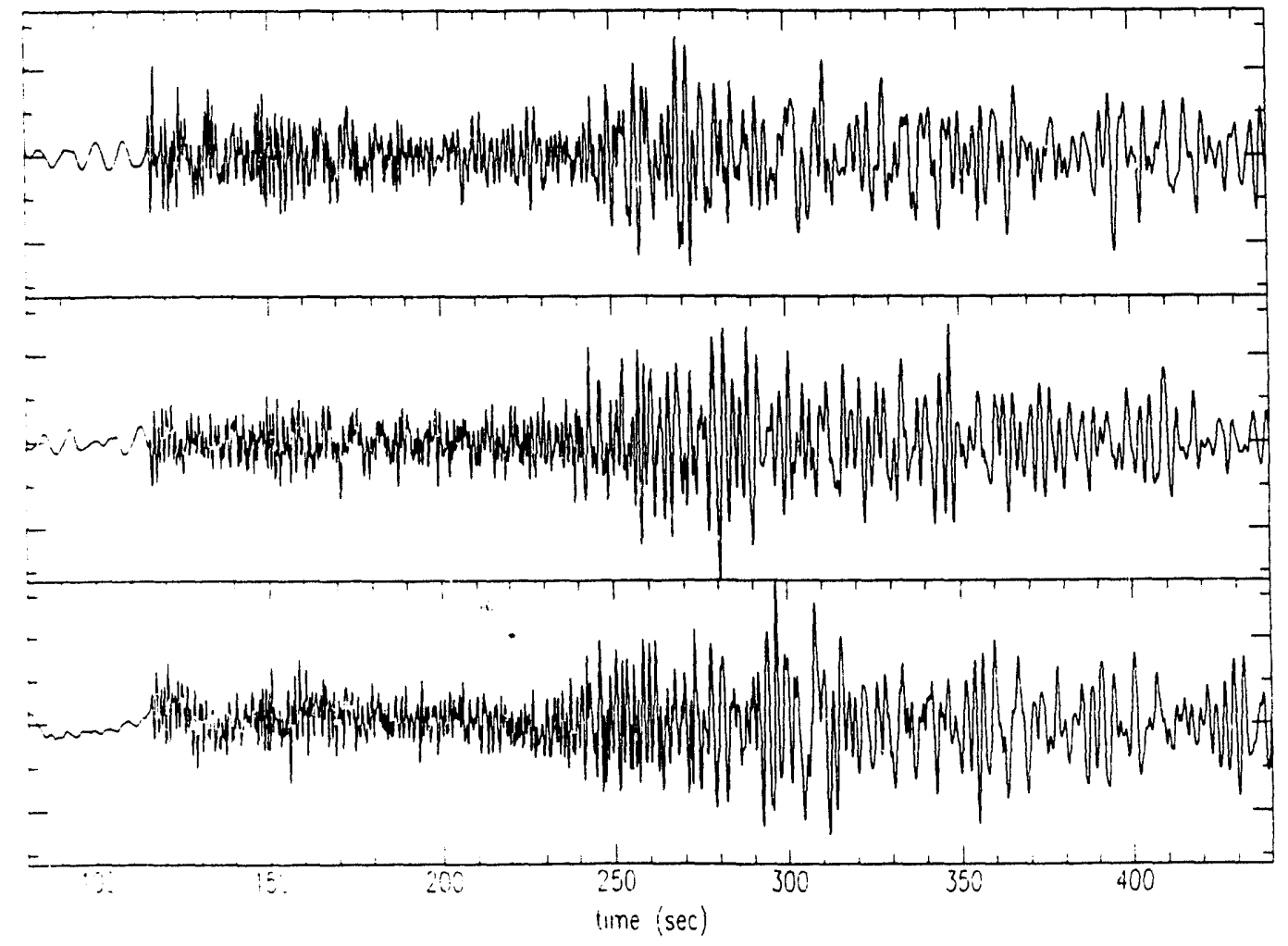

Figure 5. Unprocessed recording of Bexar on the CSMS at Pinedale. The top trace is the vertical component, the middle trace is the north component, and the bottom trace is the east component. 


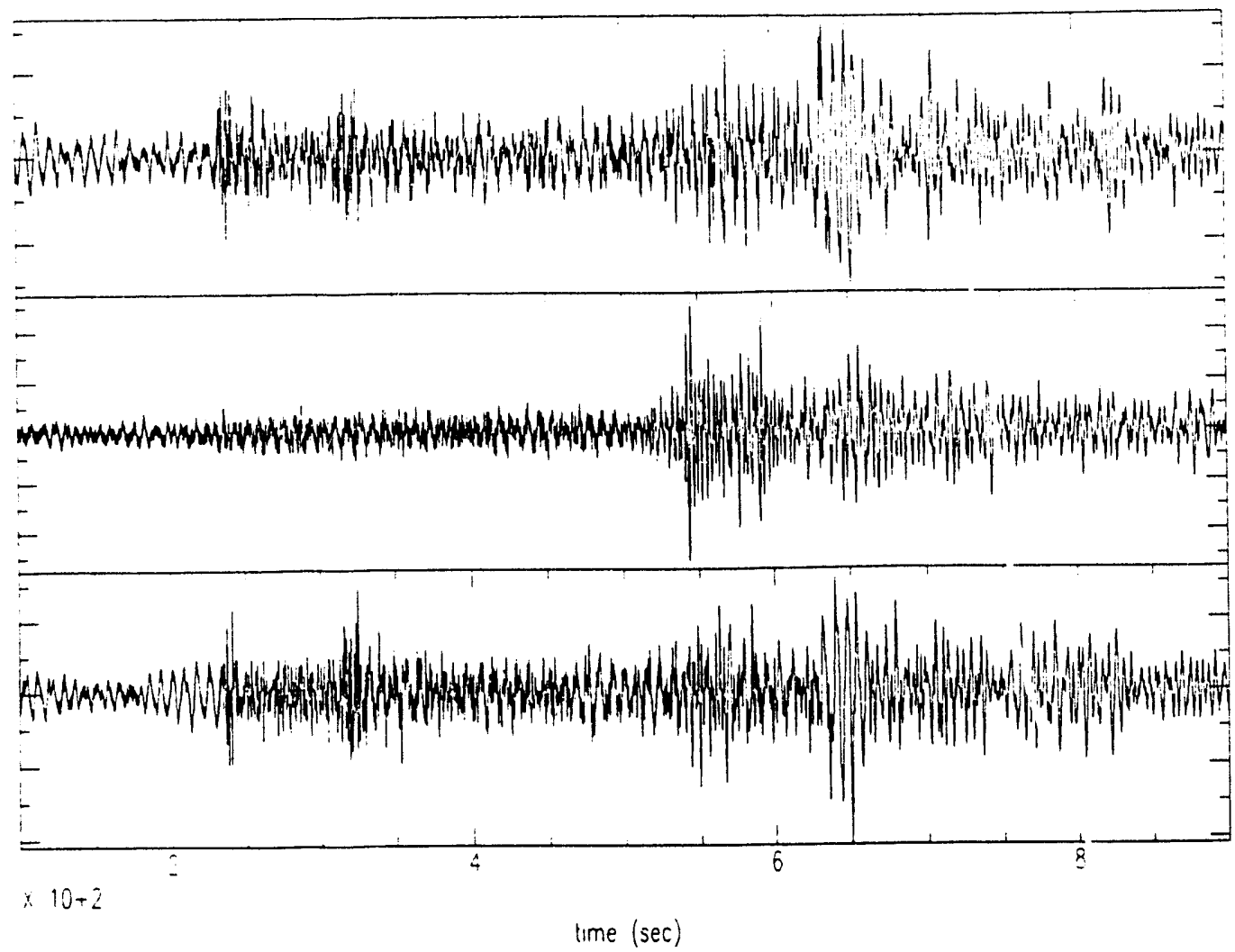

Figure 6. Unprocessed recording of Bexar on the CSMS at Tulsa. The top trace is the vertical component, middle trace is the north component, and the bottom trace is the east component.

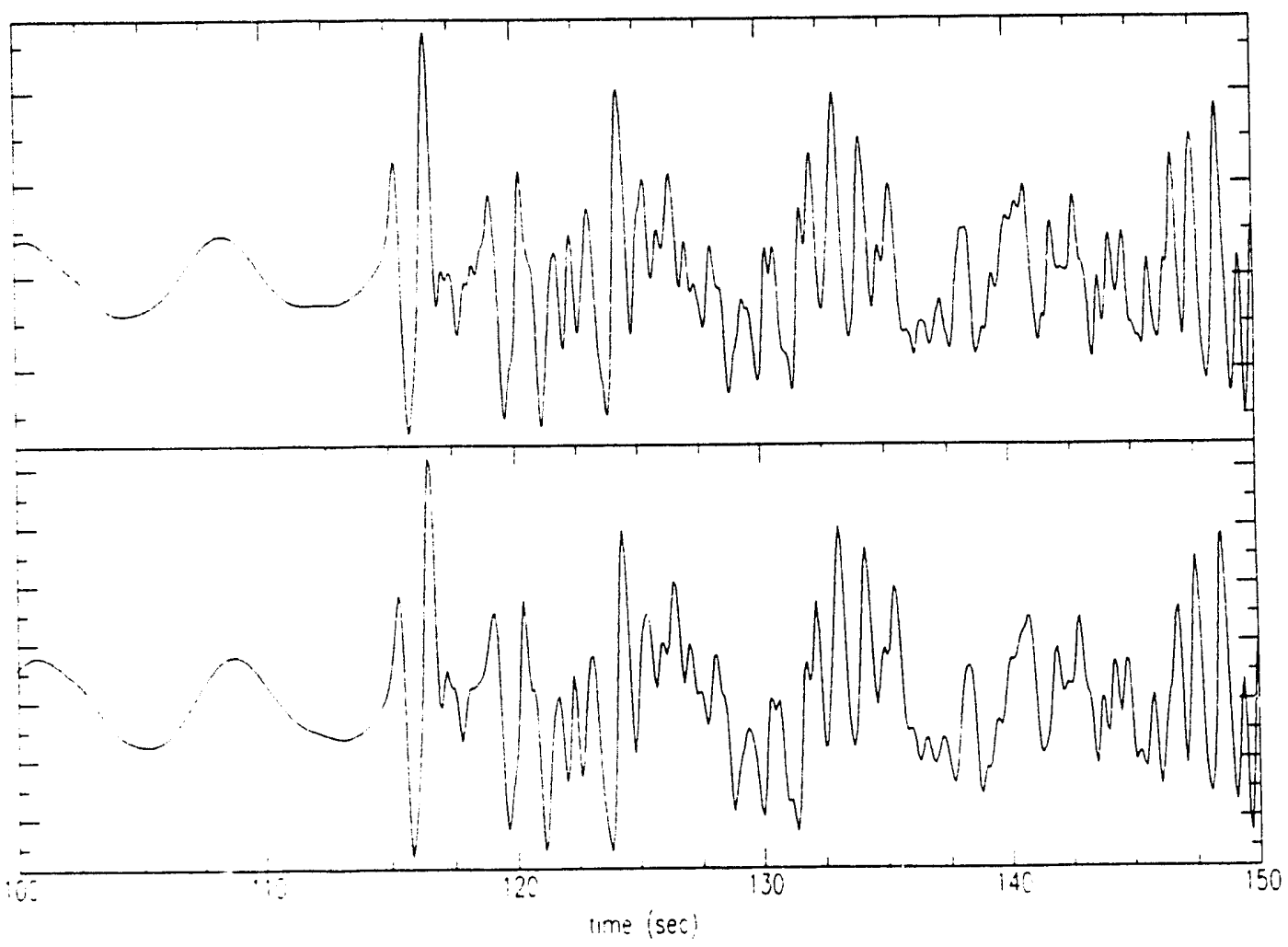

Figure 7. Comparison of the CSMS vertical component (top) and the DSVS intermediate period band vertical component (bottom) at Pinedale. 


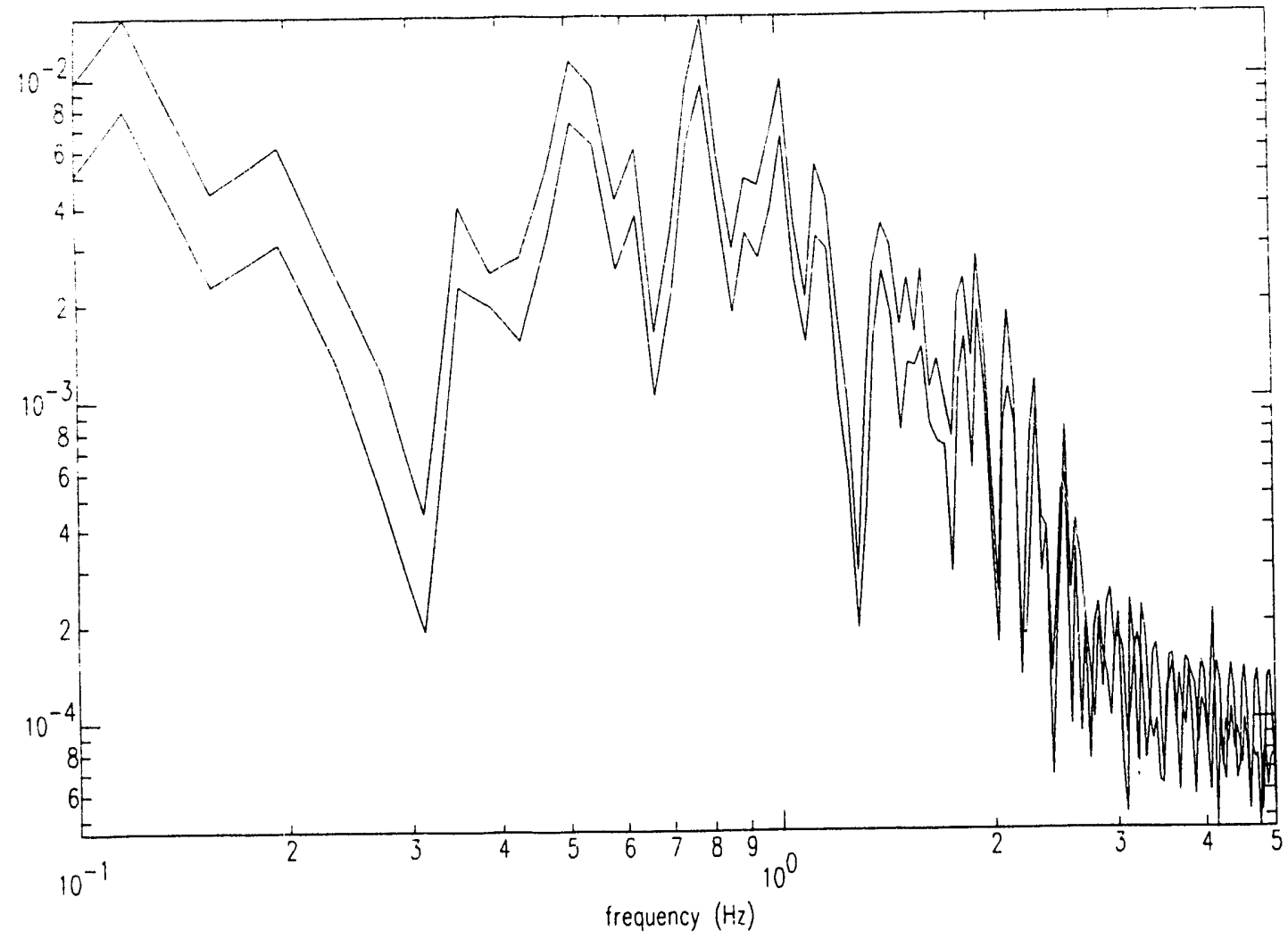

Figure 8. P-wave spectra fc $r$ the vertical component of DSVS and CSMS. 

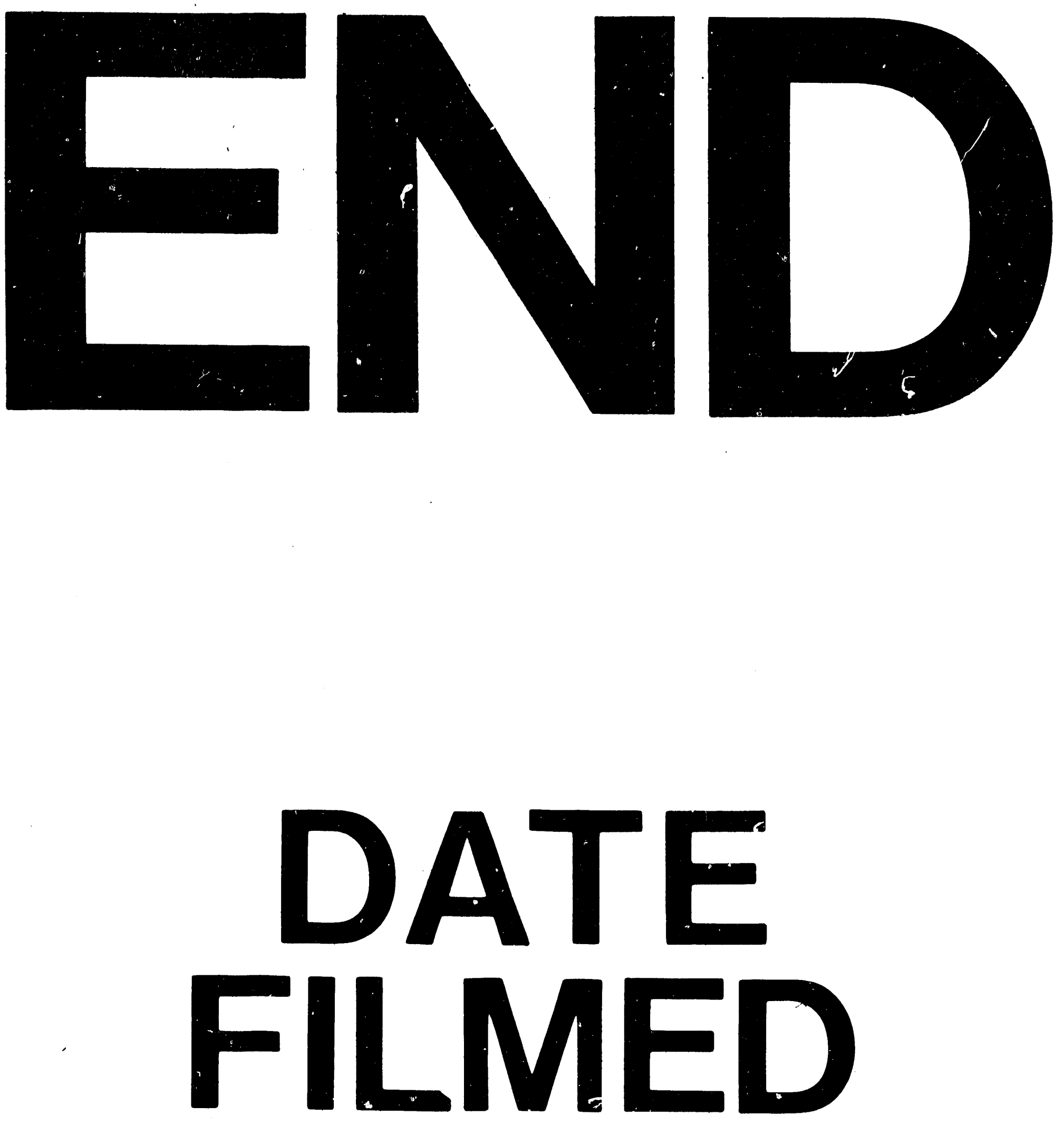

1

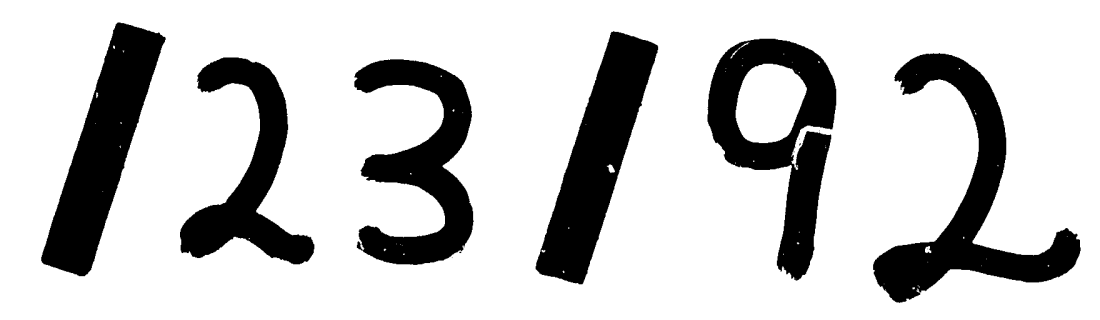


